Volume 9, No.3, May - June 2020

International Journal of Advanced Trends in Computer Science and Engineering

Available Online at http://www.warse.org/IJATCSE/static/pdf/file/ijatcse200932020.pdf

https://doi.org/10.30534/ijatcse/2020/200932020

\title{
A Grading Scale Measurement to Assess Glaucomatous Disc Damage Stages in Primary Open Angle Glaucoma
}

\author{
V.Satya Aruna ${ }^{1}$, B.Chaitanya Krishna ${ }^{2}$ \\ ${ }^{1}$ Research Scholar, Department of Computer Science \& Engineering, Koneru Lakshmaiah Education Foundation, \\ Vaddeswaram, Guntur, India. satya.aruna.v@gmail.com \\ ${ }^{2}$ Associate Professor, Department of Computer Science \& Engineering, Koneru Lakshmaiah Education \\ Foundation, Vaddeswaram, Guntur, India. chaitu2502@kluniversity.in
}

\begin{abstract}
Primary Open Angle Glaucoma develops at optic nerve head of the eye which transfers visual information from eye to brain. The gradual changes in the optic disc and cup segmentation and neuroretinal rim width are responsible for the formation of glaucomatous eyes .The dense set of optic fiber nerves act as a network system and creates Optic Nerve Head. Optic Cupping will be performed whenever few weak optic nerves dies that produces neuroretinal rim between optic disc and optic cup. The presence of thick rim in the healthy eye is common, but the gradual structure changes will appears towards glaucomatous eyes. In this paper, we study various disk damage stages like initial and advanced to assess primary open angle glaucoma with the support of the narrowest rim width location and placement. The measurement by grading scale method in various angles and locations of optic cup produce outcome to predict disc damage at initial level more accurately and also helps to prevent to enter in to advanced stage.
\end{abstract}

Key words : Optic Disc, Neuroretinal rim, Optic Cup, Open angle glaucoma, optic nerve, Glaucomatous Eyes, Sight impairment, Visual field defects

\section{INTRODUCTION}

Sight impairment as well as Visual Field defects are primary signs of chronic eye diseases like glaucoma. These problems are most common in the transformation of healthy eye to a glaucomatous eye. Visual Field information loss is the initial phase of identifying glaucoma. It should be noticed that not all visual field defects causes glaucoma only. Further clinical examination is needed to confirm glaucoma cases purely. The perfect next phase to identify is optic disc and cup segmentation using cup and disc ratio CDR and measurement of narrowest rim width located between disc and cup[1,3].
Disc and cup segmentation is common in the examination of glaucomatous eyes by almost all clinicians. It is a regular process by clinical experts checking about Intraocular Pressure and clogging of outflow of fluid in and out of the drainage canal system of the optic disc and retinal nerve fiber layers(RNFL)[2-4]. The dense set of optic nerve fibers will transmit visual information from retina to brain. These millions of optic nerves create optic nerve head (ONH). The optic disc is shaped as a bright orange vertical oval, from the huge network of the optic nerves few of the weak nerves will die inside optic nerve head and creates a cup shape which is called "optic nerve cupping". An healthy eye optic cupping is negligible or too small to compare with the optic disc and like a circle and placed at the center point of the optic disc $[3,15]$. This OC within OD creates a thick rim in the normal eye[1]. Whenever it is transforming in to defected glaucoma eye gradually the rim will become thin and some times disappear. The size of the optic cup and neuroretinal rim width both are inversely proportional. This can estimate the severity of the progression in the various glaucomatous stages starting from initial to advanced stages[1-3].

The European Glaucoma Society revealed guidelines on the size of the optic disc, and these are three types in size small disc $<1.45 \mathrm{~mm}$, average disc $1.45 \mathrm{~mm}-1.9 \mathrm{~mm}$ and finally larger $>1.9 \mathrm{~mm}$. And Spaeth in 2002 defined Disk Damage Likely hood Scale and stages with three categories "risk stage", "damage stage" and "disability stage" $[3,16]$.

The size of the optic cup is proportional to the size of the optic disc in the normal eye (ie) in large disc there is large existence of large and small disc existence of small cup. But this situation is reverse in the defected eyes[2-3]. The irregular enlargement of the optic cup overlapping on disc, displacement of cup in the disc is possible in the glaucomatous eyes. These cases finally clogs the outflow The actual problem starts in these cases, and it is necessary to of fluid and that can be identified by the absence of the rim. Finding width of the narrowest rim is to assess the present condition and glaucoma current stage accurately [13]. And most importantly identification of initial stage like "risk 
stage" will helps to prevent to enter in to the advanced stage with proper and immediate care and treatment[5]. Extensive research is taking place in disease diagnosis using Machine Learning Techniques. H.D.Gadade, D.K.Kirange have used a machine learning approach for tomato leaf disease classification[17]. Sarni Suhaila Rahim, Nur Aishah Jamal Akhshah and Shahril paruma have developed a Breast cancer self examination system using three dimension simulation [18]. Dewo Wishnu Satya Kusuma,Muhammed Akbar Rivai and Gunawan Wang have developed a medical record data history system to help doctors in identifying the dangerous diseases in patients[19]. Sathya Aruna and B.Chaitanyakrishna have developed a visual field test using correlation and association mapping for an early assessment of open angle and normal tension glaucoma[20]. [21] have developed a Diabetes prediction system using kernel based support vector machine. [22] have done a comparative study of logistic regression and SVM for the diagnosis of thyroid disfunction. In this study, we will concentrate on thinness of the rim or gradual decrease in the rim width[6,7]. Stage by stage identification in regular intervals will definitely find the problem area as early as possible. In this study, the medium and advanced stages like "glaucoma damage" and "disability" stage also be identified. All these stages identified by measuring grading scale from optic disc to cup wherever placement of the cup is taken with in the disc $[12,14]$. The necessary components are disc size, diameter of the disc and cup, optic disc, location and placement of the cup by angle, circumference of the optic disc, focal rim width, rim and disc ratio, thin and thick rim measurement, narrowest rim width.

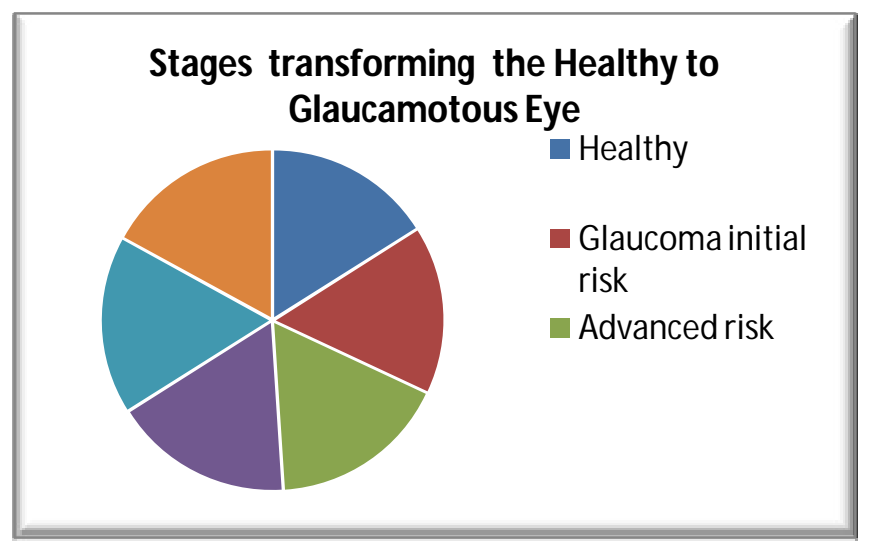

Figure 1: Six total possible stages derived from healthy eye to Glaucoma eye

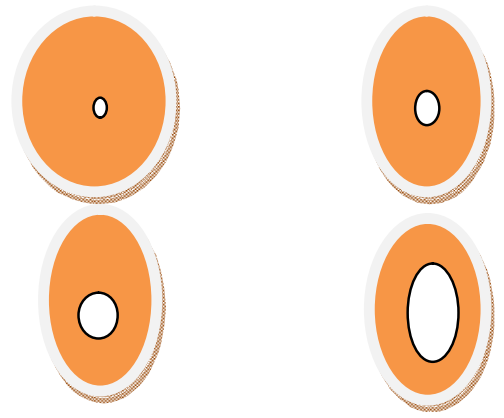

Figure 2: Optic Cup enlargement in the disk

\section{METHODOLOGY}

Vision impairment will be developed as in the increased path with the decrease in the capacity of fluid outflow ,abnormal IOP and change in the CDR[7,8]. The normal functionalities of the eye to brain nerve system will be disturbed and it will be caused by the formation of narrowest rim width or absence of rim between Optic Disc and Optic Cup [7,9]. This grading scale approach , first identify pinkish optic cup with in the bright optic cup[10,11] . At the very first observations regarding health eyes we can identify no such type of rim formation because of no clogging of fluid outflow. Then rim value will be considered as zero.

Table 1: Narrowest rim width observations to derive possible stages (Source: European Glaucoma Society)

\begin{tabular}{|c|c|c|c|c|}
\hline $\begin{array}{l}\text { Disc } \\
\text { size / } \\
\text { Stage }\end{array}$ & $\begin{array}{l}\text { Small } \\
<1.4 \mathrm{~mm}\end{array}$ & $\begin{array}{c}\text { Average } \\
1.4 \mathrm{~mm}-1 . \\
9 \mathrm{~mm}\end{array}$ & $\begin{array}{r}\text { Large } \\
>1.9 \mathrm{~mm}\end{array}$ & Stages \\
\hline 1 & $>=0.5$ & $>=0.4$ & $>=0.3$ & \multirow[b]{2}{*}{$\begin{array}{l}\text { Initial } \\
\text { risk }\end{array}$} \\
\hline 2 & $0.4-0.49$ & $0.3-0.39$ & $0.2-0.29$ & \\
\hline 3 & $0.3-0.39$ & $0.2-0.29$ & $0.1-0.9$ & \multirow[t]{2}{*}{ Adv risk } \\
\hline 4 & $0.2-0.29$ & $0.1-0.19$ & $<0.19$ & \\
\hline 5 & $0.1-0.9$ & $<0.1$ & 0 or $<45^{0}$ & \multirow[t]{2}{*}{ Damage } \\
\hline 6 & $<0.1$ & 0 or $<45^{0}$ & 0 or $46^{0}-90^{0}$ & \\
\hline 7 & 0 or $<45^{0}$ & $\begin{array}{c}0 \text { or } \\
46^{0}-90^{0}\end{array}$ & $\begin{array}{l}0 \text { or } \\
91^{0}-180^{0}\end{array}$ & \multirow{2}{*}{$\begin{array}{l}\text { Advanc } \\
\text { ed } \\
\text { damage }\end{array}$} \\
\hline 8 & $\begin{array}{l}0 \text { or } \\
46^{0}-90^{0}\end{array}$ & 0 or $91^{0}-180^{\circ}$ & $\begin{array}{l}0 \text { or } \\
180^{0-}-270^{0}\end{array}$ & \\
\hline 9 & $\begin{array}{c}0 \text { or } \\
91^{0}-180^{0} \\
\end{array}$ & $\begin{array}{l}0 \text { or } \\
180^{0-}-270^{0}\end{array}$ & 0 or $270^{\circ}$ & \multirow{2}{*}{$\begin{array}{l}\text { Disabili } \\
\text { ty }\end{array}$} \\
\hline 10 & $\begin{array}{l}0 \text { or } \\
180^{0-}-270^{0}\end{array}$ & 0 or $270^{\circ}$ & ------------ & \\
\hline
\end{tabular}

The entire process flow diagram for deriving grading scale is given by the following flowchart.

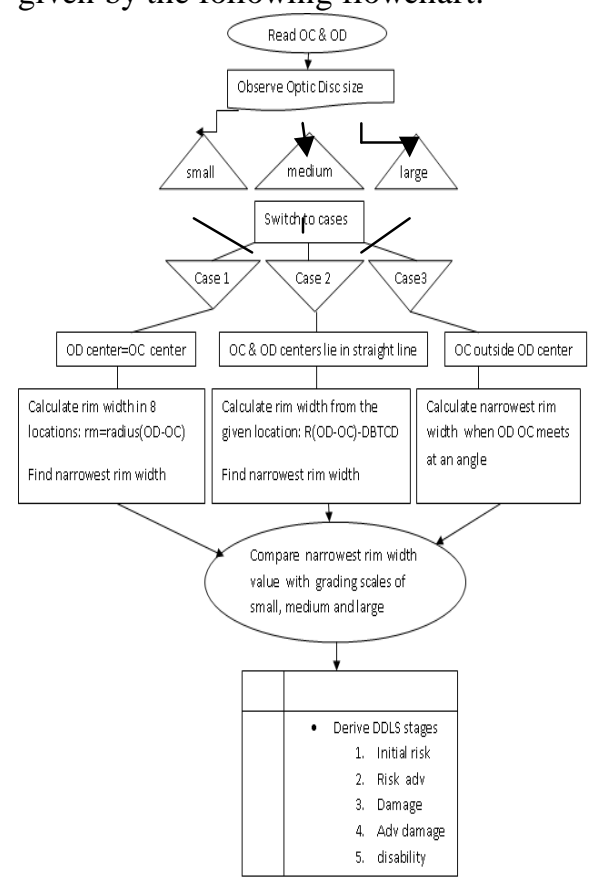


DBTCD: Distance between centers of OD and OC DDLS: Disk damage likelyhood scale

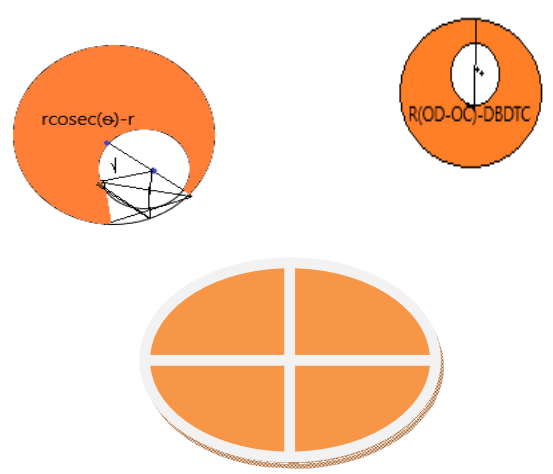

Figure 3: Optic cup inside disc for possible 3 cases

There are various components to find the rim width:

Different variables to specify rim width are $\mathrm{rm} 1, \mathrm{rm} 2, \mathrm{rm} 3, \mathrm{rm} 4, \mathrm{rm} 5, \mathrm{rm} 6, \mathrm{rm} 7, \mathrm{rm} 8$ and these 8 locations have been found from eight angles $45^{\circ}, 90^{\circ}, 135^{\circ}, 180^{\circ}, 225^{\circ}$, $270^{0}, 315^{0}, 360^{\circ}$ respectively.

fp: It is the fixed point which locate optic cup at the specified angle.

Algorithm for grading on degree of disk damage (GSD3 Scaling ) Algorithm :

Step 1: Read OD \& OC \{ divide disc area into 8 locations with different angles as mentioned in the components \}

Step 2:

Case I: Center points of OC \&OD are same.

\{Procedure for normal rim where optic cup placed at the center point of disc, Cup gradual enlargement is possible\}

Step 3: Initialize fp. \{A Fixed Point on the circumference of the optic disc\}.

Step 4: obtain locations from fp to $\mathrm{rm} 1: 45^{0}$, fp to $\mathrm{rm} 2: 90^{\circ}$, fp to $\mathrm{rm} 3: 135^{\circ}$, fp to rm $4: 180^{\circ}$, fp to rm5: $225^{\circ}$, fp to rm6: $270^{\circ}$, fp to $\mathrm{rm} 7: 315^{\circ}$, fp to $\mathrm{rm} 8: 360^{\circ}$

Step5: Initialize array $a[]=\{0,1,2,3,4,5,6,7\}$, var $\mathrm{i}=0$, init $\mathrm{j}=0$.

Step 6: Initialize $\mathrm{rm}[]=\{0,1,2,3,4,5,6,7\}$

Step 7: Loop:

Calculate radius of OD \& OC from the point $\mathrm{rm}[\mathrm{j}]$

$\mathrm{a}[\mathrm{i}]$ = radius_of_OD - radius_of_OC: rim width

Increment $\mathrm{j}$ by 1 value:

Repeat step 5 until $\mathrm{j}>7$.

Step 8: $\{$ sort array a[] $\}$

a[0]=first:

$\mathrm{i}=0$

loop

if first $>a[i]$ store first $=a[i]$ :

$\mathrm{i}=\mathrm{i}+1$ :

repeat loop until i>7 step 9: Print first . \{ var: first stores narrowest rim width with DDLS stages with grading scale \}

step 10: \{ Deriving DDLS stages with grading scale\}

1. \{ According to table 1 measurement scaling will be given \}

Case II : OC \& OD Centers and narrowest rim lie in straight line .

\{ procedure to find narrowest rim width based on cup displacement. Cup gradual displacement is possible\}

Step 11: Read OD, OC centers, radius.

Step12: Calculate DBTCD.\{distance between 2 center points

Step 13: Narrowest_rim _width= Radius (OD-OC)-DBTCD. \{Derive stages according to table 1 \}

Case III: OC located in OD at some angle.

\{Procedure to find existence of rim within the Optic Disc\}

Step 14: Calculate narrowest_rim_width $=(\operatorname{rosec} \Theta-r)$. $\{$ as shown in Figure 3$\}$ \{r: narrowest rim width $\}$ \{derive stages $\}$

\section{RESULTS}

As shown in the Table 1, the Scaling values of narrowest rim width always depends upon the size of the disc. It is clear that an optic disc of size with 3 different sizes like $1.00 \mathrm{~mm}$, $1.75 \mathrm{~mm}, 2.5 \mathrm{~mm}$ produces variations according to Table- 2 as given. The gradual decrease in the rim width obtains narrow points and risk in the stage increases. The size of the optic cup increases and rim width decreases. Both are inversely proportional.

Table 2: Assumptions for small, medium and large disc vs rim width values for various stages

\begin{tabular}{|l|l|l|l|l|l|l|}
\hline $\begin{array}{l}\text { Disc } \\
\text { size/rim } \\
\text { width mm }\end{array}$ & $\begin{array}{l}\text { Healthy } \\
\text { eye }\end{array}$ & $\begin{array}{l}\text { Initial } \\
\text { risk }\end{array}$ & $\begin{array}{l}\text { Risk } \\
\text { advan } \\
\text { ced }\end{array}$ & Damage & $\begin{array}{l}\text { Adv } \\
\text { damage }\end{array}$ & $\begin{array}{l}\text { Disabi } \\
\text { lity }\end{array}$ \\
\hline $\begin{array}{l}1.00 \mathrm{~m} \\
\mathrm{~m}\end{array}$ & 0.6 & 0.45 & 0.35 & 0.25 & 0.15 & 0.1 \\
\hline $\begin{array}{l}1.75 \mathrm{~m} \\
\mathrm{~m}\end{array}$ & 0.45 & 0.35 & 0.25 & 0.15 & 0.1 & 0.0 \\
\hline $2.5 \mathrm{~mm}$ & 0.35 & 0.25 & 0.15 & 0.1 & 0.0 & 0.0 \\
\hline
\end{tabular}

These assumption are observed between the given ranges of the disc size. In the Figure 4 given below, the bar graph visualizes the transformation of a healthy eye, Glaucomatous eye and finally disability. The important observation is, we can find the Open Angle Glaucoma at an early/primary stage is most useful for clinicians and medical experts to provide proper treatment. Our study focuses on initial stages of glaucoma for better results. 


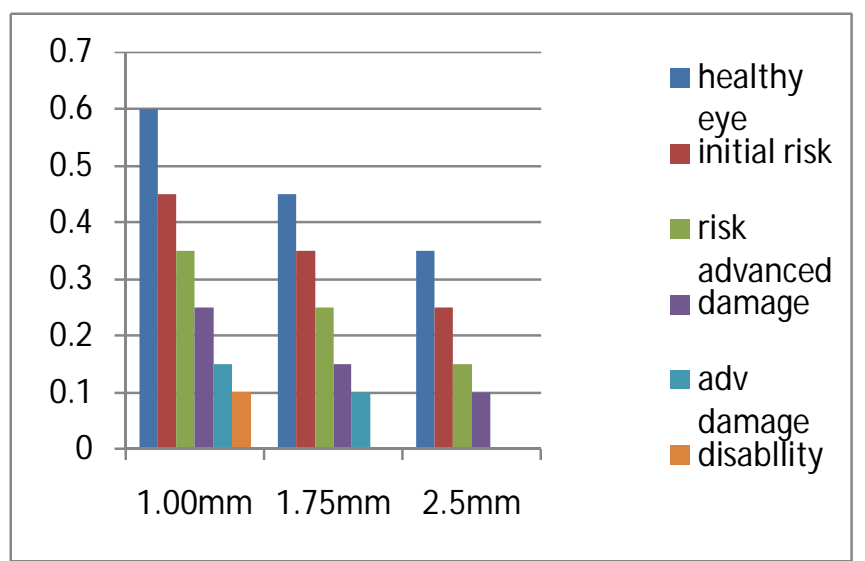

Figure 4: Chart for the Table 2 measurements to demonstrate levels of all stages $\mathrm{x}$ : disc size, $\mathrm{y}$ : narrowest rim width.

\section{DISCUSSION}

Superior neuroretinal rim is generally thicker than the nasal rim. Nasal rim is thicker than the temporal rim (ie) Inferior > Superior $>$ Nasal $>$ Temporal . The measurements above in the Table 1 are considered about disc size. It is an essential part of Glaucoma assessment and performed by Optical Coherence Tomography by clinicians in the labs. These estimations are also varied by the techniques we used.

Table 3: Assumption of rim width from

Cup to Disc Ratio

\begin{tabular}{|l|l|l|}
\hline Optic Disc & Optic Cup & Possible rim width \\
\hline Large & Small & Thick \\
\hline Medium & Large & Thin \\
\hline Medium & Medium & Normal \\
\hline Medium & Small & Thick \\
\hline Small & Large & Thin \\
\hline Small & Medium & Thin \\
\hline Small & Small & Normal \\
\hline
\end{tabular}

First of all according to disc size as shown in the Table 3 we can assume all possibilities. This approximation almost confirms about the range of risk or damage of an eye towards Glaucoma. As observed from Table 3 producing Normal and Thick rims have no risk at all. But the problem area is with on Thin rim.

From GSD3-Scaling algorithms which is based upon 3 cases. Mainly Case I works for all narrowest rim possibilities closely when cup located at center of the disc. As shown in the Figure 5 it covers two measurements in all sizes. Almost 90 percent of the inputs produces either "initial risk" or " risk advanced " states. 10 percent "damage" state occurrence as shown in the Figure 5. The measurements of Case II hardly covers initial states, due to cup displacement from the center obviously it enters in to either "damage" or "adv damage" states as shown in the Figure 6. Here in the Grading Scale algorithm Case III establishes end states also only for the confirmation. Because it produces either "advanced damage" or "disability states as shown in the Figure 7 which is out of control to respond for proper treatment. Mainly the actual focus must be on Case I and sometimes on Case II

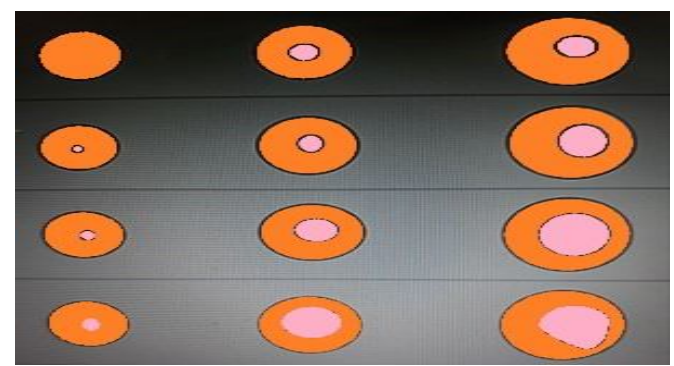

Figure 5 : Case I : Glaucoma initial and advanced risk stages demonstration. OD \& OC meets at one center point: Initial risk and advanced risk stages are demonstrated.

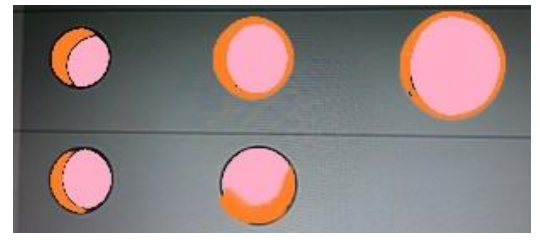

Figure 6 : Case II: When Center points of OD \& OC lies in a straight line. Glaucoma damage stage is demonstrated.

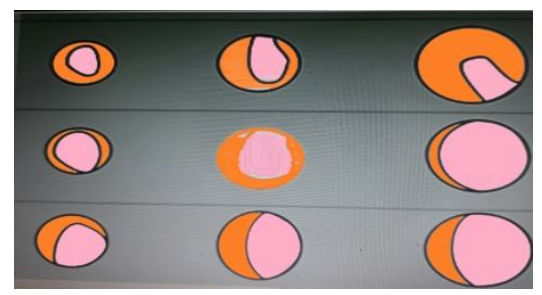

Figure 7: Case III: Glaucoma Advanced damage and disability stages are demonstrated : When OC located in OC at some angle.

\section{CONCLUSION}

In this paper, it is shown that the degree of the visual field loss will be gradually increased along with the changes in the rim width between disc and cup. The most probable cup displacements are discussed with the support of three possible cases. It is proved that, measuring narrowest rim width by mathematical grading scale approach can produce accurate results to determine DDLS stages. This approach can take part in the future work to implement an automatic model/tool for determining the Cup and Disc Ratio (CDR) to predict POAG. 


\section{REFERENCES}

1. Suha Dh. Athab, Nassir H.Selman, "Disc and Cup Segmentation for Glaucoma Detection" , Journal of mechanics of continua and mathematical sciences, 14(6), Nov-Dec 2019, PgNo: 369-383 ISSN:2454-7190 https://doi.org/10.26782/jmcms.2019.12.00026

2. Artem Sevastopolsky, "Optic Disc and Cup Segmentation Methods for Glaucoma Detection with Modification of U-Net Convolutional Neural Network", arXiv:1704.00979v1 [cs.CV] 4April 2017. https://doi.org/10.1134/S1054661817030269

3. Chunlan Yang, Min Lu Yanhua Duan and Bing LIU,"An efficient optic cup Segmentation method decreasing the influences of blood vessels", BioMedical Engineering Online (2018) ,https://doi.org/10.186/s1 2938-018-0560-y.

4. Ahmad Almazroa, Kaamran Raahemlfar et al., "Optic Disc and Cup Segmentation Methodologies for Glaucoma Image Detection:A Survey", Hindawi Publishing Corporation , Journal of Opthtalmology, Vol-2015, Article ID 180972.,28 pages, https: // dx.doi .org/10.1155/2015/180972.

5. Ahmed Almazroa, Sami Alodhayb et al., "An Automatic Image Processing System for Glaucoma Screening", Hindawi, International Journal of Biomedical Imaging", Volume 2017, Article ID4826385, 19pages, https://doi.org/10.1155/2017 14826385

6. Sripad Krishna Devalla, Khai Sing Chin et al., "A Deep Learning Approach to Digitally Stain Optical Coherence Tomography Images of the Optic Nerve Head", Glaucoma, Investigative Ophthalmology and VisualScience-2018-74. http://doi.org/10.1167 /iovs.1722617.

7. R Priyadarshini et al., "Optic Disc and Cup Segmentation in fundus retinal images using feature detection and morphological techniques", CURRENT SCIENCE,VOL 115 NO 4,25 AUGUST 2018 https://doi.org/10.18520/cs/v115/i4/748-752

8. Mohammad Salman Haleem et al., " A Novel Adaptive Deformable Model for Automated Optic Disc and Cup Segmentation to Aid Glaucoma Diagnosis" , IMAGE SIGNAL PROCESSING, J Med Syst (2018) ,https://doi,org/10.1007/s 10916-017-0859-4.

9. Madhusudhan M., Malay N., Nirmala S.R., Samerendra D. (2011) Image Processing Techniques for Glaucoma Detection. In: Abraham A., Mauri J.L., Buford J.F., Suzuki J., Thampi S.M. (eds) Advances in Computing and Communications. ACC 2011. Communications in Computer and Information Science, vol 192. Springer, Berlin, Heidelberg https://doi.org/10.1007/978-3-642-22720-2_38

10. Huazhu Fu , Jaing Llu et al., " Joint Optic Disc and Cup Segmentation Based on Multi-Label Deep Network and Polar Transformation", arXiv:1801.00926[cs.CV]
11. Kim JM, Kyung H, Shim SH, Azarbod P, Caprioli J. Location of Initial Visual Field Defects in Glaucoma and Their Modes of Deterioration. Invest Ophthalmol Vis Sci. 2015;56(13):7956-7962. doi:10.1167/iovs. 15-17297.

12. An G, Omodaka K, Hashimoto K, et al. Glaucoma Diagnosis with Machine Learning Based on Optical Coherence Tomography and Color Fundus Images. $J$ Healthc Eng. 2019; 2019:4061313. Published 2019 Feb 18. doi:10.1155/2019/4061313

13. Vasudevan et al., "Optic Disc Segmentation for Glaucoma Screening System Using Fundus Images", Clinical Ophthalmology, 2017;11:2017-2029, doi: 10.2147/OPTH.S140061

14. C51251: "Glaucoma management part-2- Optic Disc assessment in Glaucoma", Optician "Career long support for optical professionals" https://www.opticianonline.net/cet-archive/118

15. Dietter, J., Haq, W., Ivanov, I.V., Norrenberg, L.A., Völker, M., Dynowski, M., Röck, D., Ziemssen, F., Leitritz, M.A., \& Ueffing, M. (2019). Optic disc detection in the presence of strong technical artifacts. Biomed. Signal Process. Control.,53.. https://doi.org/10.1016/j.bspc.2019.04.012

16. Mohammad .U Khan, Nighat Mir et al., "Optic Disc Detection and Segmentation with Vessel Convergence and Elliptical Symmetry Evidences", Science Direct, $16^{\text {th }}$ International Learning and Technology Conference 2019 Artificial Intelligence and Machine Learning: Embedding the Intelligence, Procedia Computer Science,Vol:163 (2019) 609-617. https://doi.org $/ 10.1016 / j$.procs. 2019.12 .143

17. H.D.Gadade, D.K.Kirange , “ Machine learning approach towards tomato leaf disease classification", International Journal of Advanced Trends in Computer Science and Engineering, 9(1), 490-495, Jan-Feb(2020) https://doi.org/10.30534/ijatcse/2020/67912020

18. Sarni Suhaila Rahim, Nur Aishah Jamal Akhshal, Shahil Paruma, "Development of Breast Cancer self examination three dimensional simulation", International Journal of Advanced Trends in Computer Science and Engineering, 9(1), 579-586, Jan-Feb(2020) https://doi.org/10.30534/ijatcse/2020/80912020

19. Dewo Wishnu Setya Kusuma, Muhammad Akbar Rivai and Gunawan Wang , “ Business Intelligence Infrastructure Medical Records Data History System to help doctor in differencing rare and dangerous disease in patient", International Journal of Advanced Trends in Computer Science and Engineering, 9(1), 664-672, Jan-Feb(2020) https://doi.org/10.30534/ijatcse/2020/93912020

20. V.Sathya Aruna, B.Chaitanyakrishna, "An Early Assessment of Open Angle and Normal Tension Glaucoma based on Visual Field Test using 
Correlation and Association Mapping”, International Journal of Advanced Trends in Computer Science and Engineering, 9(2), Mar-Apr (2020)

https://doi.org/10.30534/ijatcse/2020/46922020

21. Annavarapu Naga Prathyusha and M R Narasingarao, "Diabetic Prediction Using Kernel Based Support Vector Machine", International Journal of Advanced Trends in Computer Science and Engineering, 9(2), Mar-Apr(2020)

https://doi.org/10.30534/ijatcse/2020/43922020

22. Deepthi Gurram and M R narasingarao, "A comparative study of support vector machine and logistic regression for the diagnosis of thyroid dysfunction", International Journal of Engineering and Technology, $7(1.1)(2018), 326-328$.

https://doi.org/10.14419/ijet.v7i1.1.9714 